rate through one year was $14.4 \%$ (17/118). Procedural devicerelated SAE were observed in $4.1 \%$ subjects $(5 / 121)$.

In multivariate models, independent predictors of Raymond Occlusion Class III at one-year follow-up included wide-neck aneurysm (odds ratio $[\mathrm{OR}]=5.69 ; 95 \%$ confidence intervals [CI] 1.69-19.15, $\mathrm{p}=0.0050), \quad$ balloon-assisted coiling $(\mathrm{OR}=3.81 ; 95 \%$ CI $1.28-11.37, \mathrm{p}=0.017)$, and age $\geq 65 \mathrm{yr}$ $(\mathrm{OR}=0.15 ; 95 \%$ CI $0.027-0.78, \mathrm{p}=0.025)$. Balloon-assisted coiling also predicted recanalization at one year follow-up $(\mathrm{OR}=2.54,95 \%$ CI 1.03-6.27, $\mathrm{p}=0.043)$.

Conclusion This subset analysis suggests that the SMART COIL System achieves adequate embolization in ruptured aneurysms with adequate retreatment rates over one year.

Disclosures D. Fiorella: 1; C; Penumbra, Inc. 3; C; Penumbra, Inc. B. Bohnstedt: None. C. Schirmer: None. R. Bellon: 3; C; Penumbra, Inc. R. DeLeacy: None. R. Starke: None. A. Spiotta: 1; C; Penumbra, Inc. 2; C; Penumbra, Inc.

\section{E-038 CORRELATION ANALYSIS OF ENDOVASCULAR TREATMENT FOR RUPTURED INTRACRANIAL ANEURYSM: PREDICTORS FOR UNFAVORABLE OUTCOMES AND RECURRENCE}

${ }^{1} \mathrm{~W}$ Yoon, ${ }^{1} \mathrm{~W}$ Yoon*, ${ }^{1} \mathrm{H}$ Roh, ${ }^{2} \mathrm{~S}$ Suh, ${ }^{1} \mathrm{~T}$ Kwon. ${ }^{1}$ Neurosurgery, Guro hospital, Korea university, Seoul, KOREA, REPUBLIC OF; ${ }^{2}$ Radiology, Guro hospital, Korea university, Seoul, KOREA, REPUBLIC OF

\subsection{6/neurintsurg-2019-SNIS.113}

Purpose The aim of this study is to identify significant predictors of unfavorable outcome and recurrence in the patients treated with endovascular treatment in the setting of ruptured aneurysms.

Method and material A total of 102 ruptured aneurysms of 102 patients who were treated with endovascular treatment including stent-assisted coil embolization and coil embolization without stent were enrolled in this study. In order to identify significant predictors of unfavorable outcome and recurrence, retrospective correlation analysis was performed.

Result A total of 72 patients (70.59\%) showed favorable outcome (mRS 0-2) at their last follow up day and a total of 30 patients (29.41\%) showed unfavorable outcome (mRS 3-6). On univariate and multivariate analysis for predictors of outcome in all grades SAH patients, initial Hunt-Hess grade $(p=.018)$, periprocedural complications $(p=.014)$, external ventriculostomy $(E V D)$ presence $(p=.030)$ were significant predictors of unfavorable outcome, whereas age $(p=.009)$, rebleeding before treatment $(p=.002)$, EVD presence $(p=.003)$, delayed cerebral ischemia (DCI) $(\mathrm{p}=.035)$ were significantly associated with unfavorable outcome in the patients with good grade $\mathrm{SAH}$ who were defined as initial Hunt-Hess grade 1-3. On univariate and multivariate analysis for predictors of recurrence, aneurysm volume $(\mathrm{p}=.043)$, initial Raymond classification $(\mathrm{p}=.038)$ had a significant correlation with recurrence.

Conclusion The rate of unfavorable outcome in this study of SAH patients treated with endovascular method was $29.41 \%$ and the recurrence rate was $20.59 \%$. The present study demonstrates various predictors for unfavorable outcome and recurrence in the SAH patients treated with endovascular method. Therefore, careful individualized consideration in treatment for acute SAH patients who are planned to be treated with endovascular treatment is necessary.
Disclosures W. Yoon: None. W. Yoon: None. H. Roh: None. S. Suh: None. T. Kwon: None.

\section{E-039 ENDOVASCULAR FLOW-DIVERTING STENTS FOR INTRACRANIAL ANEURYSMS}

${ }^{1} \mathrm{~A}$ Nada*, ${ }^{1} \mathrm{M}$ Brunet, ${ }^{1} \mathrm{~S}$ Chen, ${ }^{1} \mathrm{~S}$ Sur, ${ }^{1} \mathrm{O}$ Elwardany, ${ }^{1} \mathrm{G}$ Zenonos, ${ }^{1} \mathrm{~A}$ Jahmshidi, ${ }^{1} \mathrm{D}$ MccCarthy, ${ }^{1} \mathrm{D}$ Sheinberg, ${ }^{2} \mathrm{~A}$ Zaher, ${ }^{2} \mathrm{M}$ Elsherbiny, ${ }^{2} \mathrm{M}$ Kassem, ${ }^{2} \mathrm{~S}$ Serag Eldin, ${ }^{2} \mathrm{~N}$ Mansour Ali, ${ }^{2} \mathrm{~A}$ Abd Elmoneim Ezz Eldin, ${ }^{1} \mathrm{~J}$ Thomson, ${ }^{1} \mathrm{D}$ Yavagal, ${ }^{1} \mathrm{E}$ Peterson, ${ }^{1} \mathrm{R}$ Starke. ${ }^{1}$ Neurological surgery, miami university, miami, FL; ${ }^{2}$ Neurological surgery, mansoura university, Mansoura, Egypt

\subsection{6/neurintsurg-2019-SNIS.114}

Background and purpose Flow-diverting devices now offer a treatment alternative for cerebral aneurysms. The concept of these devices is to promote thrombosis of aneurysms without filling the aneurysm cavity by deviating the blood flow away from the aneurysm neck which means treatment of the diseased segment harboring the aneurysm instead of treating the aneurysm itself. We present the results of a large single-center series of patients treated with the PED, including long-term follow-up.

Materials and methods Between January 2015 and February 2019, sidewall aneurysms with a neck ( $\geq 2 \mathrm{~mm}$ to $19 \mathrm{~mm}$ ) or unfavorable dome-neck ratio $(\leq 1.5)$; large/giant, fusiform, M1 dysplastic aneurysms, dissecting, blister like, and recurrent sidewall aneurysms; aneurysms at difficult angles; and aneurysms in which a branch was originating directly from the sac were treated with the PED. Patients were pre-medicated with dual antiplatelet medications. Data, including demographics, aneurysm features, clinical presentation, complications, results, and follow-up information are presented.

Results One hundred and fourteen aneurysms in 114 patients were treated. Of these, (100\%) PEDs were used for treatment. Adjunctive coiling was performed in 73 aneurysms (64\%). Symptomatic in-construct stenosis was detected in 1 patient $(0.9 \%)$ treated with percutaneous trans-arterial angioplasty. Any event rate as (Contrast extravasation and ICH, M1 occlusion or perforation, ICA occlusion, brain stem stroke, new $\mathrm{SAH}$ at the same side of treatment, ASDH, 3rd nerve palsy, Femoral pseudo aneurysm, breaking of delivery wire, snare of microwire, worsening of the double vision) was $9 / 114(7.8 \%)$, with a permanent morbidity of $4.3 \%$ and mortality of $0.9 \%$. Control angiography was available in 108 (94.7\%) patients. The aneurysm occlusion rate was $91.2 \%$ in 6 months, increasing to $94.6 \%$ in one year.

Conclusions Flow diverters allow for the endoluminal reconstruction of a parent artery for the treatment of complex and difficult-to-treat aneurysms. There are multiple devices on the market, but the PED is the most-studied and the only device with FDA approval. Published aneurysm occlusion rates are favorable with an acceptable adverse event rate when used for approved indications.

Disclosures A. Nada: None. M. Brunet: None. S. Chen: None. S. Sur: None. O. Elwardany: None. G. Zenonos: None. A. Jahmshidi: None. D. MccCarthy: None. D. Sheinberg: None. A. Zaher: None. M. Elsherbiny: None. M. Kassem: None. S. Serag Eldin: None. N. Mansour Ali: None. A. Abd Elmoneim Ezz Eldin: None. J. Thomson: None. D. Yavagal: None. E. Peterson: None. R. Starke: None. 